\title{
Fully compressible simulations of the baroclinic instability in protoplanetary disks
}

\author{
Samuel Richard ${ }^{1, a}$ and Pierre Barge ${ }^{1, b}$ \\ ${ }^{1}$ Aix Marseille Université, CNRS, LAM (Laboratoire d'Astrophysique de Marseille) UMR 7326, 13388, \\ Marseille, France
}

\begin{abstract}
The occurrence of the baroclinic instability in protoplanetary disks is investigated using fully compressible numerical simulations. We study the role of disk stratification and heat transfer in the development of the instability. Vortices are found to form whatever the importance of the stratification but to grow and persist only when the disk is not stably stratified. In the presence of thermal diffusion the baroclinic instability leads to hollow vortices that decays into transient vortical structures.
\end{abstract}

\section{Introduction}

In this paper we study the possibility to form vortices in protoplanetary disks with the goal to explore their role in planet formation. The baroclinic instability is a well known mechanism in geophysical flows that should also play an important role in the evolution of accretion disks. This instability produces vortices through the baroclinic source term $\nabla \rho \times \nabla P$ of the vorticity equation. It is a nonlinear instability that comes into play when the disk is stratified and has a radial entropy gradient. The theoretical studies of Klahr [6] and of Johnson \& Gammie $(2005,2006)$ unsuccessfully described the mechanism. The problem was clarified by Petersen el al [7]using anelastic numerical simulations but the way to trigger the instability and its non-linear character was found by Lesur \& Papaloizou [6]. These authors have shown that vortices can grow due to a baroclinic feedback mechanism that is effective only when stratification is unstable and when heat transfer is present in the disk. In fact their work is mainly based on incompressible simulations. Here our objective is to revisit the problem using fully compressible numerical simulations.

\section{Disk model and numerical simulations}

The equations we solve are the compressible fluid equation in polar coordinates with the central potential of the star and assuming heat can be transferred in the gas. They read:

$$
\frac{\partial \rho}{\partial t}+\vec{\nabla} \cdot(\rho \vec{V})=0
$$

\footnotetext{
ae-mail: samuel.richard@oamp.fr

be-mail: pierre.barge@oamp.fr
} 

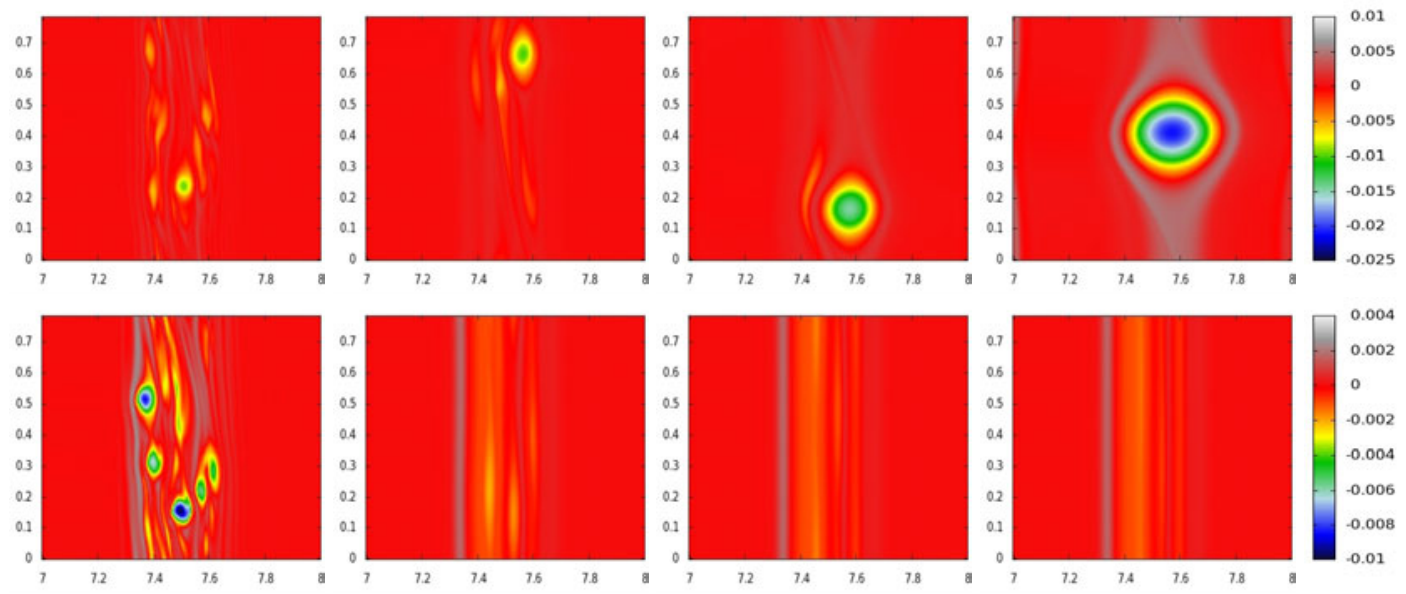

Figure 1. Vorticity map for $N^{2}<0$ (top) and $N^{2}>0$ (bottom) after 20, 200, 400 and 600 rotations, respectively

$$
\begin{gathered}
\frac{\partial \rho \vec{V}}{\partial t}+\vec{\nabla} \cdot(\rho \vec{V} \vec{V})+\vec{\nabla} P=\rho \vec{\nabla} \phi \\
\frac{\partial \rho e}{\partial t}+\vec{\nabla} \cdot((\rho e+P) \vec{V})=\rho \vec{V} \cdot \vec{\nabla} \phi+Q
\end{gathered}
$$

where $\rho$ is the density, $\vec{V}$ the velocity, $\mathrm{P}$ the pressure, $e=P / \rho(\gamma-1)+\vec{V}^{2} / 2$ the total energy and $\phi$ the gravitational potential. The gas is assumed to be perfect one and $\gamma=1$.4. Two different heat transfers are tested : (i) a thermal cooling defined by $Q=\rho\left(T-T_{0}\right) /(\gamma-1) / \tau$ where $\tau$ is a relaxation time and (ii) a thermal diffusion defined by $Q=\Delta\left(T-T_{0}\right) \gamma /(\gamma-1) / P e$ where $P e$ is the Peclet number; $T_{0}$ is the equilibrium temperature. For the background, the standard value of the density and temperature of the disk are given by decreasing power laws with index $p$ and $q$. The unperturbed flow is a steady state equilibrium with a zero radial velocity and an azimuthal velocity resulting from the balance between the star gravity and the radial pressure gradient of the gas. In non dimensional form we get : $\rho=r^{-p}$, $T=r^{-q} / \gamma M^{2}, P=\rho T, u=0, v=\sqrt{\frac{1}{r}+\frac{r}{\rho} \frac{\partial P}{\partial r}}$ with $M$ the Mach number at $1 \mathrm{AU}$.

The stratification of the disk is quantified by the Brunt-Väisälä frequency $N$, defined by $N^{2}=$ $-\frac{1}{\gamma \rho} \frac{\partial P}{\partial r} \frac{\partial}{\partial r} \ln \left(\frac{P}{\rho^{\gamma}}\right)=(p+q)(p(\gamma-1)-q) T /\left(\gamma / r^{2}\right)$. The disk is stable if $N^{2}>0$ and unstable if $N^{2}<0$. Since $p$ and $q$ can be chosen as positive numbers, the stability of the disk depends on the sign of $p(\gamma-1)-q$. So, disk stability will be discriminated following the values chosen for $p$ and $q$.

Simulations were performed with a finite volume code specifically developed for the study of protoplanetary disks [9]. High resolution is necessary to correctly simulate the development of the baroclinic instability. So, as to save computational time, our simulations are performed in a box that covers a limited region of the disk $(0<\theta<\pi / 4$ and $7 A U<r<8 A U)$ and with a resolution of $300 \times 300$ grid points.

\section{Thermal cooling}

In this section we assume heat transfer is due only to thermal cooling with a constant relaxation time ( $\tau=160$ in code unit). Two simulations were performed following the stratification of the disk is 


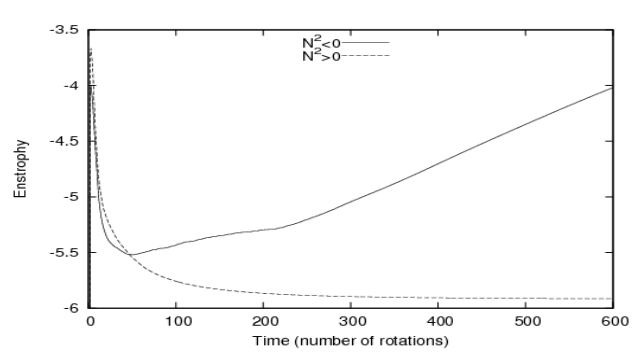

Figure 2. Evolution of the enstrophy for a stable and an unstable stratification.

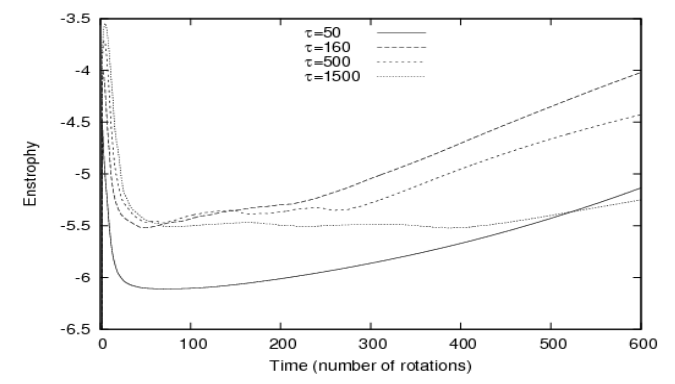

Figure 3. Evolution of the enstrophy for various cooling times.

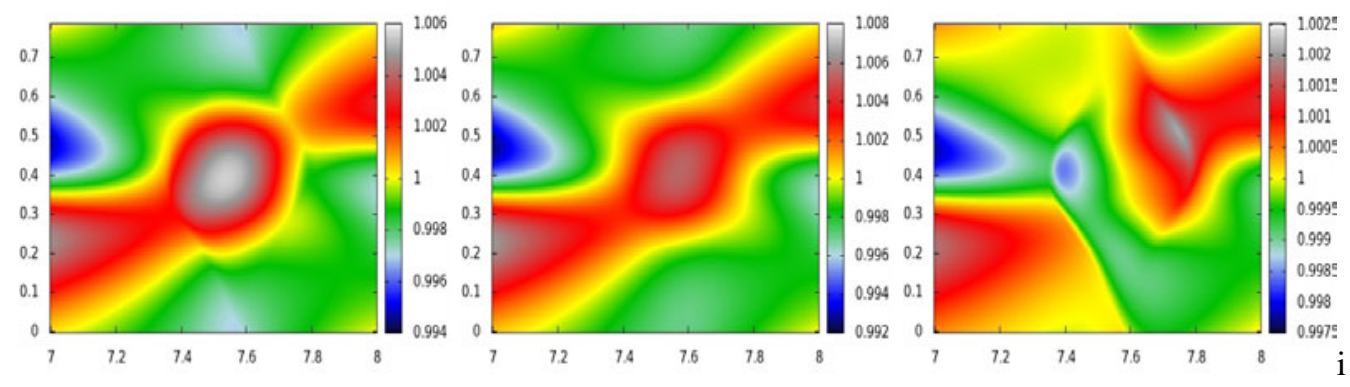

Figure 4. Structure of a baroclinic vortex after 600 rotations. From left to right: the density, the pressure and the temperature of the gas in the meridional plane, respectively

stable or not. The case $(p=1.5 ; q=0.5)$ corresponds to a stable stratification $\left(N^{2}>0\right)$. The case ( $p=1 ; q=0.5)$, corresponds to an unstable stratification $\left(N^{2}<0\right)$. The two simulations begin in the same way with the formation of vortices, but proceed differently following disk stratification is stable or not. If stratification is stable the vortices weaken and completely vanish (see bottom of figure 1). If stratification is unstable, the vortices strengthen (by the baroclinic term) and grow in successive mergings; a single big vortex remains after a few hundred rotations (see top of Fig.1). Figure 2 shows the evolution of the enstrophy in the two cases: it is increasing only if the stratification is unstable.

The structure of a baroclinic vortex is presented on figure 4: density and pressure have a bumpy shape whereas temperature has a contrasted extent with a large hot region outward and a cooler region inward. The spatial repartition of the temperature is consistent with the instability mechanism described by Lesur \& Papaloizou (2010) : fluid particles moving on a loop are thermalized along azimuthal excursions and accelerated by buoyancy forces along radial excursions.

For unstable stratifications, the role of thermal cooling was tested for different values of $\tau$ ranging from 50 to 1500. The baroclinic instability can develop in each case but time evolutions are different (cf figures 3 and 6). Large values of $\tau$ make easier vortex formation but tends to inhibit growth; on the other hand small values of $\tau$ slow down vortex formation and speed up growth. 

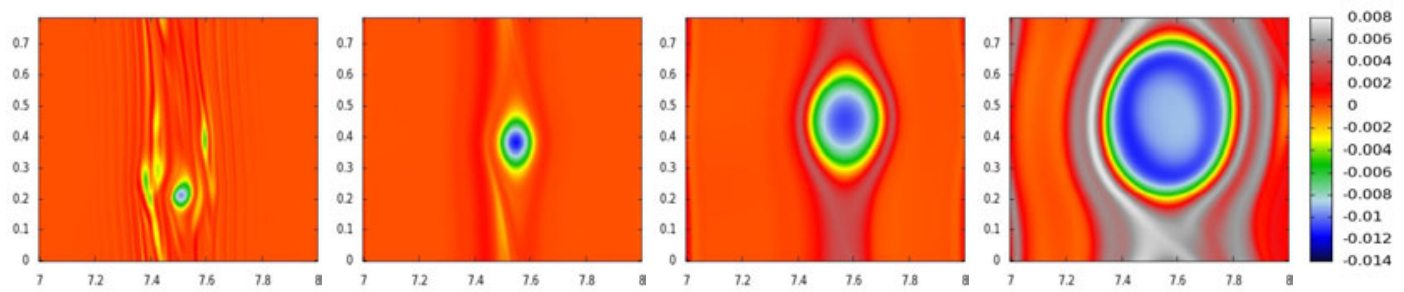

Figure 5. Vorticity map for a disk with thermal diffusion after 20, 200, 500 and 1600 rotations, from left to right, respectively. The formed vortex becomes a hollow vortex.

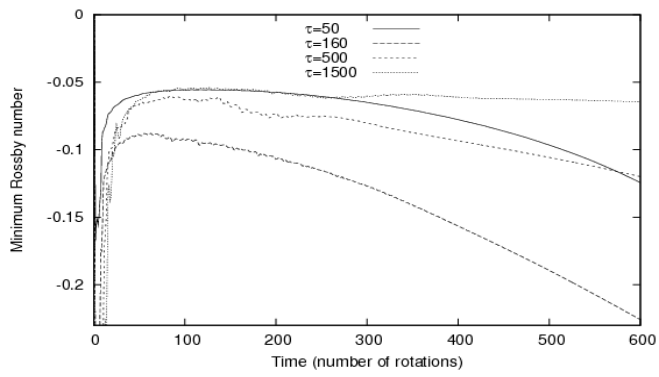

Figure 6. Evolution of the minimum of the Rossby number for different values of the cooling time.

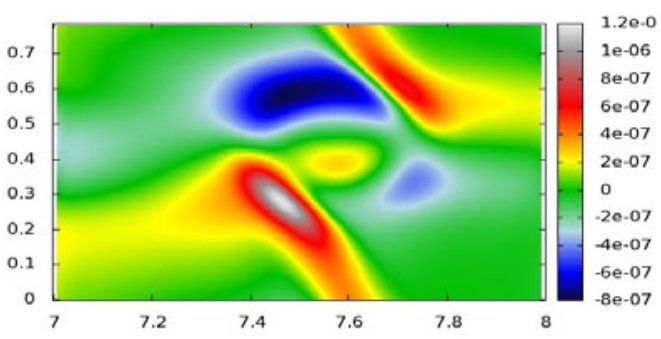

Figure 7. The Baroclinic term after 500 rotations for a disk with thermal diffusion.

\section{Thermal diffusion}

In this section we assume heat transfer is due only to thermal diffusion. A simulation was performed in similar conditions as in the previous section. Assuming unstable stratification small vortices begin to form; they grow and merge like in the case of thermal cooling. However, after a few hundred rotations, the situation is changed: vorticity increases at the vortex center while it decreases at the vortex edge. We finally obtain a hollow vortex for which the vorticity is localized not in the center of the vortex but at the edge (figure 1). This vortex structure is due to the form of the baroclinic term which is positive in the center of the vortex (figure 7).

\section{References}

[1] Johnson, B. M. \& Gammie, C. ApJ 626, 978 (2005a)

[2] Johnson, B. M. \& Gammie, C. ApJ 635, 149 (2005b)

[3] Johnson, B. M. \& Gammie, C. ApJ 636, 63 (2006)

[4] Lesur G. \& Papaloizou J. C. B., A\&A 513, A60 (2010)

[5] Klahr, H. H., \& Bodenheimer, P. ApJ 582, 869 (2003)

[6] Klahr, H. ApJ 606, 1070 (2004)

[7] Petersen, M.R., Julien, K., \& Stewart, G.R. ApJ 658, 1236 (2007a)

[8] Petersen, M.R., Stewart, G.R., \& Julien, K. ApJ 658, 1252 (2007b)

[9] Richard S. \& Barge P., EpJ web of conference, this volume 